Caballero (y Pilar Góngora), deben ser vistos, y así los recomendamos a nuestros alumnos o lectores a quienes le importen las letras bellas españolas y las cuestiones morales que plantea la guerra civil de 1936, cuestiones a las que ningún ciudadano creemos que debería resultar ajeno.

\section{Francisco ABAD Nebot}

CAmPs, Assumpta: El Decadentismo italiano en la literatura catalana, Berna, Peter Lang AG, International Academic Publishers, 2010, págs. 355.

Assumpta Camps, docente di Filología italiana presso l'Università di Barcellona, è autrice di numerosi libri, tra i quali, ricordiamo: La recepción de Gabriele D'Annunzio en Cataluña (19961999) in due volumi, La traducción (1998), La recepción literaria (2002), Italia-España en la época contemporánea (2009). Ha curato, inoltre, tra gli altri, i seguenti volumi: Ética y política de la traducción en la época contemporanea (2004) Traducción, (sub)versión, transcreación (2005), Traducción y diferencia (2006), Traducción e interculturalidad (2008) e La traducción literaria en la época contemporánea (2008).

La risposta straordinariamente concorde della coscienza letteraria del primo Novecento alla decadenza oggettiva dei valori e delle istituzioni del mondo borghese costituiscono l'essenza del Decadentismo italiano. In questa stagione della cultura europea assurgono a livello emblematico le vicende intellettuali e l'opera di Svevo, Pirandello e D'Annunzio. Proprio quest'ultimo, con minuzia di particolari e precisione scientifica, è oggetto d'interesse della poliedrica Camps nell'ultimo decennio del secolo scorso. Una ri- cerca continua e costante nel tempo che porta la studiosa all'inizio degli anni Dieci al presente saggio.

El Decadentismo italiano en la literatura catalana descrive l'influenza e la presenza che il Decadentismo italiano ha esercitato sulla letteratura catalana, dalla fine del XIX secolo sino alla fine della prima guerra mondiale con brevi considerazioni che si estendono sino agli anni Venti, frutto, in gran parte, dell'enorme interesse e, addirittura, amore che la saggista ha per le due letterature romanze: 1'italiana e la catalana. In questo senso, quest'opera sistematizza in maniera definitiva la ricca e complessa trama della questione nel suo insieme, celebrando lo straordinario apporto creativo della studiosa al panorama della letteratura italiana, catalana ed europea tout court.

$\mathrm{Nel}$ periodo che va dalla crisi agraria (1880) all'inizio della prima guerra mondiale (1914) il ceto intellettuale fornisce le risposte più rappresentative ai processi indotti dalla svolta capitalistica nella nostra società attraverso la mediazione di una generale denuncia di una crisi dei valori culturali tradizionali, degli statuti teorici ottocenteschi e dello stesso rapporto con la società. E, concorde con Leone De Castris, «altresì attraverso la mediazione di un inquieto confronto con il clima critico e ribelle della cultura europea contemporanea, già più avanzata nell'elaborazione di simili risposte perché già precedentemente sollecitata dalle contraddizioni di uno sviluppo capitalistico più articolato e maturo».

L'analisi della ricezione critica del Decadentismo italiano nel contesto iberico della Catalogna segue l'ordine cronologico delle interpretazioni che generò il suo arrivo. Assumpta Camps, con l'intento riuscito di non trascurare nessun aspetto critico e letterario, articola la sua riflessione in 
sei parti (Los inicios, Vitalismo y regeneracionismo, El Programa culturalista, Teatro poético, teatro nacional, Eugeni D'Ors, Latinismo y conflicto bélico) precedute da una premessa chiarificatrice e di profonda meditazione.

Partendo da un comparativismo tradizionale e dall'ottica italiana -afferma lucidamente l'autrice- l'analisi portata a termine si mostra inefficace quando, di fatto, si tratta di procedere in modo contrario. Lo studio delle ricezione del Decadentismo italiano in Catalogna nel cambio di secolo si orienta, al contrario, all'analisi del contesto di ricezione. In linea generale, si può considerare che non esiste un approccio comparativista della ricezione. Dunque, studiare la ricezione di D'Annunzio nel contesto del Modernisme catalano, per esempio, non si allontana molto dall'analisi della ricezione attuale di D'Annunzio in Italia, se si eccettuano le traduzioni e le introduzioni, di elementi nuovi, più o meno forti, nel sistema letterario e culturale, in generale, di ricezione, che conviene valutare nella loro giusta misura.

L'inizio di detta ricezione, concepito come un'anteprima interpretativa dei seguenti capitoli, si può situare sulla rivista L'Avenç nell'ultimo trimestre del 1884 ad opera di Ramón D. Perés. Passando per i debutti teatrali a Barcellona e per le recensioni su La Vanguardia, nel volume si analizzano le più importanti letture del Decadentismo italiano nell'ambito catalano che si producono principalmente dentro la corrente del Vitalismo e del Rigenerazionismo, del Grupo de Reus e delle riviste Catalonia e Joventut alla fine del secolo XIX, e del programma del Culturalismo, nel primo decennio del secolo XX. Il programma culturalista, d'accordo con Vicenç Llorca i Berrocal, recupera il Modernisme con l'elaborazione di un piano rigeneratore per trasformare la società attraverso la cultura. La tradizione viene ora interpretata in termini di futuro, in modo che si sintetizza un linguaggio nuovo, moderno e rivolto alla collaborazione in favore della creazione di una cultura nazionale. Questa è una caratteristica molto importante che differenzia il Modernisme catalano da altri movimenti esteticamente simili, ma privi di questo obiettivo. $\mathrm{E}$ in questo senso si distinguerà anche dal Modernisme letterario proprio della letteratura castigliana.

Sono le riviste El Poble Català con i suoi autori Gabriel Alomar, Diego Ruiz, Manuel de Montoliu con il controverso artículo del 1908 La Nova Plèiade (che dará il nome al gruppo), Jeroní Zanné, Pere Prat i Gaballí, Artur Martínez i Serinyà, Alfons Maseras, Ramón Vinyes, Vincenç Solé de Sojo e Alexandre Plana che recepiscono il Decadentismo italiano e che dimostrano la passione della saggista nello scavare alla ricerca delle comuni radici, inteso come un processo che rende la letteratura ricevente più cosciente della propria identità. Assumpta Camps con dinamismo illustra e giustifica ciascuna delle sue affermazioni traendo esempi dal corpus narrativo e poetico dannunziano, una per una, certosinamente documentata in nota. Meritano particolare rilievo le estensioni del Modernisme nella provincia, l'autrice, infatti, non dimentica e dedica la sua attenzione al Grupo de Girona ed in particolare a Joseph Tharrats. L'attenzione si focalizza anche su Valencia nonostante il movimento sia un riflesso dell'ambiente culturale barcellonese.

Il saggio ci fa altresì riflettere sull'influenza che esercitò il Decadentismo nella formulazione di un teatro poetico nazionale in Catalogna. In effetti, D'Annuzio costituisce un punto di riferimento nella drammaturgia europea di fine secolo, come modello per il teatro poetico che si 
contempla come la via per superarare tanto il teatro borghese come il teatro realista. Si propone, dunque, come modello di teatro dell'arte, specialmente, per il suo intento di recupero della tragedia greca, ed in concreto, per la sua riformulazione in chiave moderna, del fatum, in una concezione drammatica che si concepisce come cerimonia civile. Allo stesso tempo, la studiosa è capace di catturare il cambio di tendenza che costituisce l'arrivo del Noucentisme nel panorama culturale catalano e come questo arrivo abbia influito nella ricezione posteriore del Decadentismo italiano, per concludere con l'analisi di detta ricezione negli anni della guerra. Le differenze tra Modernisme e Noucentisme non sono così radicali come a volte si è voluto far credere. In effetti, i seguaci del Noucentisme respinsero gli atteggiamenti ribelli e decadenti e si proposero una stretta collaborazione con i politici del momento. Eugeni d'Ors, il principale teorico del movimento, strutturò l'ideologia del Noucentisme e si contrappose alla posizione di esemplarità espressa dagli scrittori della La Nova Plèiade e così, Gabriele D'Annunzio, secondo la studiosa, si converte «en un punto de referencia innegable para él, aunque cargado de connotaciones negativas».

Assumpta Camps dimostra, in piena sintonia con l'autorevole De Castris, come gli intellettuali del Decadentismo hanno lavorato in definitiva in contesti determinati e tutti ricostruibili attraverso il loro lavoro, che è sempre stato parte attiva e qualificante di quei contesti in un tempo assai preciso e innovativo della storia europea. "Questi processi nuovi intaccano profondamente il vecchio status della cultura: e in ogni opera del Decadentismo, questo impatto è a suo modo segnato, è il referente di una rivolta che diventa ricerca, critica, produzione di forme».
SALVATORE BARTOLOTTA

Espín Templado, Ma Pilar, La escena española en el umbral de la modernidad (Estudios sobre el teatro del siglo XIX), Valencia, Tirant Humanidades, 2011.

Al hojear La escena española en el umbral de la modernidad de $\mathrm{M}^{\mathrm{a}}$ Pilar Espín Templado, vienen a mi memoria unas palabras que Emilia Pardo Bazán estampó en 1888, en el prólogo a De mi tierra, una obra en la que, como en el caso que nos ocupa, recopiló trabajos suyos de muy diversa índole, unidos, sin embargo, por un hilo conductor. La escritora deseaba desde hacía tiempo publicar uno de los discursos que integran ese volumen, pero no de forma independiente, sino acompañado de otros textos hasta formar un libro, «pues aborrezco -afirmaba- los folletitos semejantes a obleas, que no hay forma de encuadernar y que en todas las bibliotecas estorban»».

No es que las separatas estorben, pero tenemos experiencia de que no siempre aparecen en el momento en que se necesitan. De ahí la utilidad de volúmenes como éste, en el que una especialista, buena conocedora de una materia que ha investigado durante años, pone a disposición de otros estudiosos una serie de trabajos con cierta homogeneidad temática, como son estos dieciséis Estudios sobre el teatro del siglo XIX, que en su origen eran dieciocho, pues los capítulos 11 y 15 están respectivamente formados por dos trabajos previos, ahora unidos.

La mayor parte de los textos del volumen vieron la luz en revistas como Anales de Literatura Española, Epos o Cuadernos del Lazarillo, en homenajes a maestros y colegas como José Fradejas o Amelia García-Valdecasas, o fueron presentados en congresos internacionales celebrados en Nápoles (Italia), Saluzzo (Italia), Lille 